\title{
The general profile of children and adolescents with major depression referred to the Free State Psychiatric Complex
}

F J W Calitz, BA Hons, MA (Clin Psychol), DPhil

M Veitch, Bsc Hons, MSC (Clin Psychol)

A Verkhovsky, Medical Student

D Nieuwoudt, Medical Student

J Myburg, Medical Student

Department of Psychiatry, University of the Free State, Bloemfontein

\section{G Joubert, BA, BSC}

Department of Biostatistics, University of the Free State

Background. Depression can have far-reaching effects on the functioning and adjustment of young people. Among both children and adolescents depression confers an increased risk for illness, interpersonal and psychosocial difficulties.

Objective. The aim of this study was to determine the profile of children (birth - 12 years) and adolescents (13- 18 years) with major depression referred to the Child Mental Health Unit at the Free State Psychiatric Complex (FSPC) from 1 January 2004 to 31 December 2004

Method. A total of 904 children and adolescents were referred to the Unit during 2004. From these referrals 100 children and adolescents (1 1.06\%) were diagnosed with major depression and were included in the study.

Data analysis. The data were summarised using frequencies and percentages (categorical variables) and means of percentiles (numerical variables).

Results. The majority $(74 \%)$ of the cases were in the adolescent age group and $61 \%$ were girls. The main complaints presented by the parents or in the referral letter by the general practitioners were behavioural problems (37\%) such as stealing, aggression and attention-seeking behaviour, followed by decrease in school performance (25\%), suspected depression $(21 \%)$, suicidal tendencies $(17 \%)$, and school refusal $(8 \%)$. Twelve per cent of the group had lost a family member. The main presenting clinical symptoms of major depression in the study group were sleep disturbance (59\%), dysphoric (depressed) mood (57\%), disturbance in appetite (49\%), constant fatigue/loss of energy $(43 \%)$, inability to enjoy activities that would normally give pleasure $(37 \%)$, and impaired concentration (36\%). Somatic complaints occurred in $42 \%$ of the study population.
Most of the complaints involved headaches $(28 \%)$, stomach aches $(14 \%)$, neck and back pain $(8 \%)$ and nausea $(6 \%)$. Thirty-three per cent of the participants with physical complaints were on medication. Behavioural problems present among the children/adolescents included fighting (26\%), disobedience $(23 \%)$, staying away from school (15\%), stealing (13\%), telling lies (13\%), running away from home (8\%), and aggression (4\%). Fifty-six per cent $(56 \%)$ of the participants showed a decline in school perfomance and $4 \%$ had enuresis.

Conclusion. Of particular interest was the finding that the clinical features in this South African community are consistent with samples studied in First-World countries. These areas or symptoms are sleep disturbance, depressed mood, disturbance in appetite, constant fatigue or loss of energy, inability to enjoy activities that would normally give pleasure, impaired concentration and suicidal thoughts. Other notable clinical features were behaviour problems, substance abuse and somatic complaints. There is no doubt that this research will also encourage other extrapolations of First-World findings such as treatment safety and efficacy of major depression in children and adolescents.

Depression can have far-reaching effects on the functioning and adjustment of young people. In both children and adolescents depression confers an increased risk of illness, and interpersonal and psychosocial difficulties. Unfortunately, depression often goes unrecognised by families and physicians alike. Signs of depression in young people are viewed as normal mood swings typical of a particular developmental stage. In addition, health care professionals may be reluctant to prematurely 'label' a young person with a mental illness diagnosis. Yet early diagnosis and treatment of depression are critical to healthy emotional, social and behavioural development.

According to the Diagnostic and Statistical Manual of Mental Disorders (DSM-IM), diagnostic criteria for a major depressive episode, major depression in children/adolescents can be defined as significant distress or impairment manifested by 5 or more of the following criteria occurring almost daily for 2 weeks or more: (i) depressed/irritable mood; (ii) recurrent thoughts of death and suicidal ideation; 
(iii) diminished interest or pleasure; (iv) weight loss/gain - or failure to gain weight expected; ( $v$ ) psychomotor agitation/retardation; (vi) fatigue or energy loss; (vii) feelings of worthlessness, low self-esteem; (viii) diminished ability to think/concentrate; and (ix) insomnia or hypersomnia. ${ }^{2-4}$

A depressed/irritable mood or diminished interest/pleasure must be among the criteria and must represent a change from normal and previous behaviour. Some children also experience physical problems such as stomachaches and headaches that seem to have no general medical cause. In adolescents depression may also be associated with poor academic performance, social dysfunction, substance abuse, suicide attempts, and completed suicide. ${ }^{5-7}$

The prevalence of depression in the USA is $0.9 \%$ in preschool children, $2 \%$ in school-age children and 5 - $9 \%$ in adolescents. In childhood the sex ratio is equal but after puberty the ratio changes to $2: 1$, with more girls suffering from depression. ${ }^{8,9}$ Forty-five per cent of adolescents with major depression relapse in young adulthood. ${ }^{10}$

The aetiology of major depression is multifactorial and results from a combination of genetic, temperamental and environmental risk factors." Risk factors associated with the development of depression in children/adolescents are: (i) chronic general medical illness; (ii) a family history of mental illness or suicide; (iii) abuse (physical, emotional or sexual); and (iv) loss of a parent at an early age as a result of death, divorce, abandonment, or the break-up of a romantic relationship. ${ }^{12}$

Dysfunctional family relationships characterised by excessive parental criticism, lack of parental support and warmth as well as parental rejection and hostility, family and marital dysfunction and poor parent-child communication are all associated with depression. ${ }^{13-16}$

Depression is a major cause of morbidity and mortality among adolescents. It has been found that depression has the strongest independent effect on self-harm and attempted suicide. ${ }^{17-19}$ Suicide remains the 3rd leading cause of death in older adolescents and the 5th leading cause of death in children between 5 and 14 years of age in the USA and some parts of Europe. ${ }^{17}$ According to Schlebusch, ${ }^{20}$ hanging is the most frequently used method, following by shooting (35\%) and poisoning (9.8\%) across all age groups. Methods employed in non-fatal suicides among children and adolescents were mostly overdose of painkillers, benzodiazepines and antidepressants, or self-poisoning.
In the USA and Scandinavia the prevalence of suicide attempts has been found to be higher in females than males. The most common method of completed suicide in children and adolescents was the use of firearms, accounting for about two-thirds of all suicides in boys and almost one-half of suicides in girls. The second most common method of suicide in boys, occurring in about $25 \%$ of all cases, is hanging. About $25 \%$ of girls commit suicide thorough ingestion of toxic substances. ${ }^{20,21}$

Additional risk factors for adolescent suicide include a family history of suicidal behaviour, exposure to family violence, impulsivity, substance abuse and availability of lethal methods.

It is important to be aware that children with depressed mood are likely to have co-existing psychiatric disorders such as anxiety disorders, disruptive behaviour disorders, attention deficit hyperactivity disorder, learning problems, dysthymic disorder and alcohol or other substance abuse. ${ }^{22}$

In South Africa there is limited recently conducted research available on depressed children/adolescents. For this reason there was a need to determine the demographic profile, clinical presentation and possible factors associated with major depression in children.

\section{Objectives}

The aim of this study was to determine the profile of children (birth - 12 years) and adolescents (13 - 18 years) with major depression referred to the Child Mental Health Unit at the Free State Psychiatric Complex (FSPC) from 1 January 2004 to 31 December 2004.

Specific objectives were to determine: (i) the demographic profile of depressed children and adolescents; (ii) symptoms present during the disorder; and (iii) possible factors associated with major depression.

\section{Methods}

\section{Study design}

A descriptive, retrospective study was undertaken.

\section{Study population}

A total of 904 children (from birth to 12 years) and adolescents (13 - 18 years) were referred to the Child Mental Health Unit at the FSPC during 2004. From these referrals 100 children and 
adolescents (11.06\%) were diagnosed with major depression and were included in the study. The diagnoses were made by the multiprofessional team at the Unit. The team included a child and adolescent psychiatrist, a clinical psychologist, a registrar, a clinical psychology intern, psychiatric professional nurses, a social worker and an occupational therapist.

\section{Measurements}

A data form was compiled and used to enter the relevant information from the clinical files of the children and adolescents. The researchers themselves completed the data forms from the contents of the children's/adolescents' files.

\section{Data analysis}

The data were summarised using frequencies and percentages (categorial variables) and means or percentiles (numerical variables).

\section{Ethical approval}

The study was approved by the Ethics Committee, Faculty of Health Sciences, University of the Free State. Permission was obtained from the Chief Executive Officer of the FSPC.

\section{Results and discussion}

\section{Demographic information}

The majority (74\%) of the cases were in the adolescent age group. The youngest patient/subject was 6 years old and the eldest was 17 years old, with 14 being the median age. The majority (61\%) were girls. This finding is consistent with the findings of Elliot and Smiga ${ }^{8}$ and Yorbik et al. ${ }^{9}$

It was also found that 33\% of the parents of the depressed group were still married, 29\% were divorced, 16\% were single parents, 13\% were deceased, 5\% were separated, 3\% were remarried and 1\% were living together. The high divorce rate is an indication of the dysfunctional marital relationships of the parents of these chidren and adolescents with major depression. This finding is consistent with numerous findings by researchers such as Harrington, ${ }^{13}$ Herring and Kaslow, ${ }^{14}$ Zahn-Waxler et al. ${ }^{15}$ and Brennan et al. ${ }^{16}$

\section{Clinical presentation}

The main presenting clinical symptoms of major depression in the study group were sleep disturbance (59\%), dysphoric (depressed) mood $(57 \%)$, disturbance in appetite $(49 \%)$, constant fatigue/loss of energy (43\%), inability to enjoy activities that would normally give pleasure (37\%), and impaired concentration (36\%).
The main complaints presented by the parents or in the referral letter by the general practitioners were behavioural problems $(37 \%)$ such as stealing, aggression and attention-seeking behaviour, followed by decrease in school performance (25\%), suspected depression (21\%), suicidal tendencies (17\%) and school refusal (8\%). Twelve per cent of the group had lost a family member. Eleven per cent were victims of sexual, physical or emotional abuse (including rape), $6 \%$ came from families with severe financial problems, $3 \%$ had enuresis and $1 \%$ experienced family violence. A possible explanation for the unexpectedly low figure for family violence could be the following: (i) the participants could have interpreted family violence as physical violence; (ii) participants could have avoided mentioning family violence because they felt embarrassed; and (iii) the study group consisted only of those children and adolescents referred to the FSPC.

Twenty-four per cent of the participants had made suicide attempts. Of these, 58.8\% had made more than one attempt. The majority of subjects who attempted suicide were girls. Groholt et al. ${ }^{20}$ and Gould et al. ${ }^{21}$ found that in the USA and Scandinavia females had a greater prevalence of suicide attempts than males, but males were more likely to be successful in their attempt. Most of the attempts involved overdosing with a toxic substance $(62.5 \%)$, followed by hanging (16.7\%) and shooting (4.6\%) (Table I). Nevid et al. ${ }^{3}$ also found that men were more likely to use overtly violent methods, whereas women preferred less violent means such as self-poisoning by overdose. Major depression seems to be a major risk factor for suicide attempts and eventual suicide, so if depression can be diagnosed early deaths might be avoided. ${ }^{17,18}$ Forty-four per cent of the study group reported suicidal thoughts among their symptoms, 17 participants were referred because of suicidal tendencies, and 24 had at least one previous suicide attempt.

\begin{tabular}{lcc} 
Table I. Suicide attempts $(\mathbf{N}=\mathbf{2 4})$ & \\
\hline Method & $N$ & $\%$ \\
\hline Overdose & 15 & 62.5 \\
Hanging & 4 & 16.7 \\
Shooting & 1 & 4.6 \\
\hline
\end{tabular}

Somatic complaints occurred in $42 \%$ of the study population. Most of the complaints involved headaches (28\%), stomach aches (14\%), neck and back pain (8\%) and nausea (6\%). Thirtythree per cent of the participants with physical complaints were on medication.

Behavioural problems present among the children/adolescents included fighting (26\%), disobedience $(23 \%)$, staying away from 
school (15\%), stealing (13\%), telling lies (13\%), running away from home $(8 \%)$, and aggression (4\%). Fiffy-six per cent $(56 \%)$ of the participants showed a decline in school perfomance and $4 \%$ had enuresis.

The following habits were noted among the depressed patients: nail biting $(24 \%)$, smoking (18\%), thumb sucking $(3 \%)$ and alcohol use (3\%) (Table II).

\begin{tabular}{lll}
\multicolumn{2}{l}{ Table II. Habits noted among participants } \\
\hline Habits & N & $\%$ \\
\hline Nail biting & 24 & 24 \\
Smoking & 16 & 18 \\
Thumb sucking & 3 & 3 \\
Alcohol use & 3 & 3 \\
\hline
\end{tabular}

Co-morbidity was noted in $45 \%$ of the participants, viz. posttraumatic stress disorder $(22.2 \%)$, generalised anxiety disorder (15.6\%) and conduct disorder (8.9\%). This finding is consistent with the international literature, which suggests that depression is not uncommon in children who have other psychiatric disorders, such as disruptive behaviour disorders, anxiety disorders, or attention deficit/hyperactivity disorder or in children with learning problems or those who abuse substances.

Thirty-five per cent of the children/adolescents referred for depression were on antidepressant medication, or had been treated with antidepressants in the past.

\section{Factors contributing to the development of major depression}

Risk factors associated with developing depression include family history of mental illness. ${ }^{12}$ In $25 \%$ of the participants in this study it was found that there was a family history of depression, mainly affecting the mother $(84 \%$ of the $25 \%)$.

Regarding substance abuse in children and adolescents with major depression, the study revealed that only $11 \%$ of the study population had abused some kind of substance. Substances used were alcohol, dagga, 'glue', cocaine and heroin, with small differences between these substances. It is possible that some participants (child and/or parent/s) were not entirely honest about the abuse of substances and therefore the percentage may have been higher.

An interesting finding was the misuse of aspirin by 1 of the participants. From the study the frequency of aspirin intake was not clear. Although aspirin is not a drug of addiction, regular abuse of aspirin can become a prevalent bad habit. Aspirin is no longer recommended for children (under the age of 10), since if they have certain infections it may be linked to the onset of a rare and dangerous condition known as Reye's syndrome. Overdose of aspirin can also bring on dizziness, mental confusion and bleeding. ${ }^{24}$ The main ingredient in aspirin is salicylic acid, found in willow tree bark.

In the case of $35 \%$ of the participants, one or both parents abused some form of substance. In most cases (about 70\%) it was the father who abused substances. It has been shown that substance abuse by parents is a factor contributing to dysfunctional family relations and a risk factor contributing towards developing depression. ${ }^{13 \cdot 16}$

The most common stressors associated with the development of depression were family relationship problems $(48 \%)$, loss of a family member $(31 \%)$, and failing a grade (22\%).

\section{Conclusion}

The purpose of the study was to determine demographic, clinical presentation and possible factors contributing to major depression in children and adolescents. Of particular interest was the finding that the clinical features in this South African community are consistent with samples studied in First-World countries. These areas or symptoms are sleep disturbance, depressed mood, disturbance in appetite, constant fatigue or loss of energy, inability to enioy activities that would normally give pleasure, impaired concentration and suicidal thoughts. Other notable clinical features were behaviour problems, substance abuse and somatic complaints.

There is no doubt that this research will also encourage other extrapolations of First-World findings such as treatment safety and efficacy of major depression in children and adolescents li.e. if it looks like a duck and sounds like a duck it will hopefully respond like a duck to known duck medicines).

The main established risk factors prevalent in our sample were stressors, family history of depression, dysfunctional parental marital relationships and substance abuse by the parents. The use of knowing these areas of known risk is that they provide potential points of entry to interrupt the causal pathway that ends in suicide or chronic mental ill health. These points of entry can be family interventions, substance abuse programmes for kids and adults, education around the storage/easy accessibility of means of self 
harm, the need for support systems for traumatised or bereaved children and teens, and the possibility of detecting mental health problems by monitoring school performance.

Although the present study revealed significant findings, the results should be interpreted with great care, especially as far as generalisation is concerned. For example, only children and adolescents referred to the FSPC were included in the study. Another limitation is that no rating scales for depression were used and therefore no inter-rater reliability is available. However the signifcance of the findings should not be underestimated - they not only contribute to important academic data in a field that has been largely neglected in South Africa, but also provide information on demographic profile, clinical presentation and possible factors contributing to major depression in children and adolescents.

\section{References}

1. Birmaher B, Brent DA, Benson RS. Summary of the practise parameters for the treatment of children and adolescents with depressive disorders. J Am Acad Child Adolesc Psychiatry 1998; 37: 1234-1238.

2. American Psychiatric Association. Diagnostic and Statistical Manual of Mental Disorders. 4th ed. Washington: APA, 1994

3. Nevid JS, Rathus SA, Green B. Abnormal Psychology in a Changing World. New York: Prentice Hall, 2003.

4. Kaplan HL, Sadock BJ. Comprehensive Textbook of Psychiatry. 8th ed. Baltimore: Williams \& Wilkins, 1998

5. Pedersen S. Assessing and treating the depressed child. Practitioner 2002; 244 252-257.

6. Bhatia SK, Bhatia SC. Childhood and adolescent depression. Am Fam Physician 2007; 75: 73-80, 83-84

7. Hazell P. Depression in children and adolescents. Am Fam Physician 2003; 67: 577 579
8. Elliot GR, Smiga S. Depression in the child and adolescent. Pediatr Clin North Am 2003; 50: 1093-1 106

9. Yorbik O, Birmaher B, Axelson D, Williamson DE, Ryan ND. Clinical characteristics of depressive symptoms in children and adolescents with major depressive disorder. J Clin Psychiatry 2004; 65: 1654-1659.

10. Olfson M, Gemeroff MJ, Marcus SC, Waslick BD. Outpatient treatment of child and adolescent depression in the United States. Arch Gen Psychiatry 2003; 60: 1236 1242.

11. Beatson J, Taryan S. Predisposition to depression: the role of attachment. Aust NZ J Psychiatry 2003; 37: 219-225

12. Birmaher $B$, Williamson DE, Dahl RE, et al. Clinical presentation and course of depression in youth: Does onset in childhood differ from onset in adolescence. J Am Acad Child Adolesc Psychiatry 2004; 43(1): 63-70.

13. Harrington RC. Family genetic findings in child and adolescent depressive disorders. Int Rev Psychiatry 1996; 8: 355-368

14. Herring M, Kaslow NJ. Depression and attachment in famillies: A child-focused perspective. Fam Process 2002; 41: 494-518

15. Zahn-Waxler C, Klimes-Dougan B, Slattery MJ. Internalizing problems of childhood and adolescence: Prospects, piffalls, and progress in understanding the development of anxiety and depression. Dev Psychopathol 2000; 12: 443-446.

16. Brennan PA, Le Brocque R, Hammen C. Maternal depression, parent-child relationships, and resilient outcomes in adolescence. J Am Acad Child Adolesc Psychiatry 2003; 42: 1469-1477.

17. Pfeffer CR. Suicide in mood disordered children and adolescents. Child Adolesc Psychiatr Clin N Am 2002; 11 : 639-648.

18. March J, Silva S, Petrycki S, et al. The Treatment for Adolescents with Depression Study (TADS): Demographic and clinical characteristics. J Am Acad Child Adolesc Psychiatry 2005; 44(1): 28-40

19. Merry S, McDowell H, Wild Cl, et al. A randomized placebo-controlled trial of a school-based depression prevention program. J Am Acad Child Adolesc Psychiatry 2004; 43: 538-547

20. Schlebusch L. Suicidal Behaviour in South Africa. Pietermaritzburg: University of KwaZulu-Natal Press, 2005

21. Groholt B, Ekeberg O, Wichstrom L, et al. Young suicide attempters: A comparision between a clinical and an epidemiological sample. I Am Acad Child Adolesc Psychiatry 2000; 39: 868-875

22. Gould MS, Greenberg T, Velting DM, Shaffer D. Youth suicide risk and preventive interventions: A review of the past 10 years. J Am Acad Child Adolesc Psychiatry 2003 42: 386-403

23. Angold A, Costello EJ. Depressive comorbidity in children and adolescents: empirical, theoretical and methodological issues. Am J Psychiatry 1993; 150: 1779-1791.

24. Abbot FV, Fraser MI. Use and abuse of over-the counter analgesic agent. J Psychiatry Neurosci 1998; 23(1): 13-34. 